\title{
Voltage regulation and power losses reduction in a wind farm integrated MV distribution network
}

\author{
Ghaeth Fandi ${ }^{*}$, Famous Omar Igbinovia*, \\ Josef Tlusty $^{* *}$, Rateb Mahmoud ${ }^{* * *}$
}

\begin{abstract}
A medium-voltage (MV) wind production system is proposed in this paper. The system applies a medium-voltage permanent magnet synchronous generator (PMSG) as well as MV interconnection and distribution networks. The simulation scheme of an existing commercial electric-power system (Case A) and a proposed wind farm with a gearless PMSG insulated gate bipolar transistor (IGBT) power electronics converter scheme (Case B) is compared. The analyses carried out in MATLAB/Simulink environment shows an enhanced voltage profile and reduced power losses, thus, efficiency in installed IGBT power electronics devices in the wind farm. The resulting wind energy transformation scheme is a simple and controllable medium voltage application since it is not restrained by the IGBT power electronics voltage source converter (VSC) arrangement. Active and reactive power control is made possible with the aid of the gearless PMSG IGBT power converters.
\end{abstract}

K e y w o r d s: reactive power regulation, wind farm, IGBT power converters, permanent magnet synchronous generator, wind production, medium voltage distribution network

\section{Introduction}

In general, wind power has demonstrated to be the most rapidly increasing renewable power source and its enormous spread in interconnected networks is steadily bringing new problems for the electrical power system engineers. Dealing with the technological developments and the upgrading specifications of electric-power firms, an appropriate system for reactive power control in wind farms is obligatory. The structure comprises of a supervisory reactive power control and compensation system which connects various components of the wind farm in order to quantify and regulate the reactive power produced from the wind energy system $[1,2]$.

Before now, wind energy did not have any important influence on energy network regulation, but at present due to its proportion, wind energy must perform a more substantial functional role in electric-power grid performance and control. The device utilized in wind farms were initially established on squirrel-cage induction generator connected exactly to the electric-power grid. Power pulsating nature in wind energy systems were nearly straight away conveyed to the electrical grid by utilizing squirrelcage induction generator as its speed is fixed owing to its restricted slip limit. Moreover, no dynamic control of the active and reactive power occurred excepting for not many capacitor banks which assured unity power factor at the point of common coupling (PCC). As the energy ability of wind farms expands, controlling the frequency and the voltage in the electric-power grid became more important, hence the need for advance power electronics like IGBT Flexible Alternating Current Transmission System (FACTS) devices [3-6], as a brilliant interface connecting the wind energy system and the electricpower grid. IGBT FACTS Power electronics devices is altering the fundamental attributes of wind turbine from actually a power source to essentially a reactive power source for the electric-power grid. Owing to the devices utilized in wind farms, the cost per $\mathrm{kW}$ of recently developed wind energy plant is now commensurate and indeed lower than coal energy systems; consequently, applications with IGBT power electronics devices are extremely appealing [7-15]. Due to the rapid increasing ability and more notable effects to the electric-power grid network by wind farms, MV power electronic converters utilized in wind energy systems have transformed considerably [11]. In the past, power electronics converters in wind farms were principally soft starters utilized to originally interconnect squirrel-cage induction generator with electric-power grid network, and solely uncompli-

\footnotetext{
* Department of Electrical Power Engineering, Faculty of Electrical Engineering, ${ }^{* *}$ Faculty of Biomedical Engineering, Czech Technical University in Prague, Czech Technical University in Prague, CZ, fandigha@fel.cvut.cz, igbinfam@fel.cvut.cz, tlusty@fbmi.cvut.cz,

*** Department of Energy Committee, Syrian Engineers Syndicate SY, tmaa99@hotmail.com
} 
cated thyristors were employed and are not required to transmit power steadily [16]. Subsequently power electronic converters were mostly utilized for the regulation of rotor resistance of wound-rotor induction generator, where additional state-of-the-art diode bridges with chopper were utilized to regulate the rotor resistance of generators [17], specifically at nominal power performance to lessen machine-like stress and loading. Not long ago, additional modern back-to-back (BTB) power converters were instituted in huge proportion to control the energy produced from wind farms. Foremost almost entirely in the partial-scale power proficiency for the doubly fed induction generator (DFIG), subsequently in the full-scale power ability for the asynchronous (induction) generator (AG) and synchronous generator (SG) [12, 18]. By instituting the BTB power converters (PCs), it is conceivable to completely control the produced reactive power from wind farms, and as well supply additional services to the electric-power grid. Power electronics converters progressively became more and more sophisticated and brought in remarkable performance advancement for the wind energy industry, not exclusively decreasing the machine-like stress and progressively its power in a growing manner, nevertheless it further permits the entire wind farm to act like a totally regulatory production unit allowed to substantially more suitably integrate wind energy system into the electric-power grid network [16, 18].

In electric-power lines, reactive power sustains the voltage level of power lines so that active power can flow to do beneficial tasks and this is the reason reactive power is compensated by external method. It is extremely greatly essential for power line to function, and for active power to flow. This is usually achieved with the aid of a capacitor connected in parallel to electricpower transmission lines to provide reactive power to improve voltage profile or using an inductor to assimilate reactive power in order to reduce voltage profile. Today, power electronic compensators are utilized for automatic and varied level of compensation. With regards to power losses, core or no-load losses are normal distribution losses, and do not rise or reduce with adjustments in load. They are mostly affected by the features of the steel laminations utilized to produce the core of transformers. As loads level rises, the cable and wires becomes hotter, the component turn out to be more resistive, and power line losses rises. For this explanation, resistive losses rise exponentially with the current on power lines. At low-load times, the electric-power system losses are virtually altogether core losses. Throughout the time of peak electricpower demand times, anyhow, resistive losses come to be most prominent. That being the case, electric-power line loss minimizing is to a certain extent a consequence of power system design and structure, even so it is equally terribly influenced by effect of the underlying electrical loads and by how suitably maximum loads are administered. Reactive power compensation is mostly successful when executed not far from the loads connected, so that the leading current does not have an impact on wiring upstream from the end-user. Utility authorities may effectively accomplish meaningful potential advantage and reductions in power line losses by tackling both commercial and residential reactive power problems by prudently placing wind farm stations on distribution circuits.

Modern progression in the wind energy industry is a significant test for its development. The variation of wind give rise to variations in the power delivered by wind farms to the electricity network. Going by this reason, the evolution of schemes to ameliorate voltage stability, frequency stability, power quality and losses [24] is a significant area of research in the wind energy industry. Hence, this paper proposes a simulation scheme of a commercial electric-power system and a wind farm with gearless variable speed PMSG insulated gate bipolar transistor (IGBT) power converters connected to a medium voltage (MV) distribution network. The proposed grid systems are compared in terms of power losses, and voltage stability. Results for the proposed scheme at different loads were assessed showing similar efficiency gains compared to the benchmark commercial electric power grid system. Results for the various cases are presented, depicting that integration of the wind farm on the grid network has enhanced active and reactive power control to achieve better voltage profile and reduction in power losses.

\section{The benchmark commercial electric-power generation system}

This part discusses a typical scheme of the commercial electric-power generation system. This is chosen as a benchmark system against which to generate a Simulation scheme of the commercial electric-power system. Figure 1 illustrates the electrical installation network of the electric-power generation system showing the major system components. The network consists of a 90 MVA sub-transmission station connected with a 3-phase 3-line $66 \mathrm{kV}$ power line, each of the line is a 3-phase system of varying lengths on which the same installed load capacity of $20.88 \mathrm{MVA}$ is powered via a $66 / 20 \mathrm{kV}$ transformer. The length of each of the MV power lines, $L_{1}, L_{2}$ and $L_{3}$ are $50 \mathrm{~km}, 55 \mathrm{~km}$ and $60 \mathrm{~km}$ respectively.

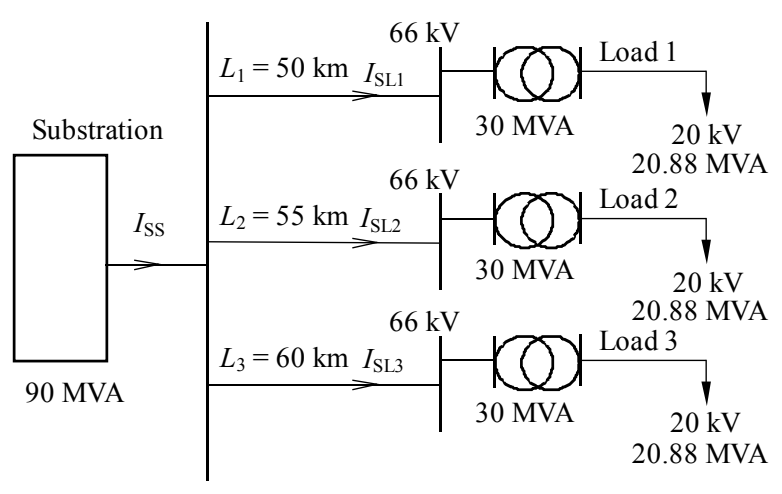

Fig. 1. The benchmark commercial electrical network 
Table 1. Parameters of the MV commercial electrical power lines

\begin{tabular}{lccc}
\hline Line number & 1 & 2 & 3 \\
\hline$R_{0}(\Omega / \mathrm{km}$ & 0.1153 & 0.413 & 0.413 \\
$L_{0}(\mathrm{mH} / \mathrm{km})$ & 1.05 & 1.05 & 1.05 \\
$L_{00}(\mathrm{mH} / \mathrm{km})$ & 3.32 & 3.32 & 3.32 \\
$C_{0}(\mathrm{nF} / \mathrm{km})$ & 11.33 & 11.33 & 11.33 \\
$C_{00}(\mathrm{nF} / \mathrm{km})$ & 5.01 & 5.01 & 5.01 \\
Frequency $(\mathrm{Hz})$ & 50 & 50 & 50 \\
Length $(\mathrm{km})$ & 50 & 55 & 60 \\
Phase resistance $\Omega$ & 5.8 & 6.34 & 6.92 \\
$X \Omega(16.5$ & 18.2 & 20 \\
$B \mu \mathrm{S}$ & 1.78 & 1.96 & 2.1 \\
\hline
\end{tabular}

Table 2. Parameters of the three-phase $66 / 20 \mathrm{kV}$ transformers $1,2,3$

\begin{tabular}{lcc}
\hline Transformer & LV side & HV side \\
\hline Connection & D11 & Yg \\
$V_{\text {rms }}(\mathrm{kV})$ & 66 & 20 \\
$R(\mathrm{~m} \Omega)$ & 653 & 20 \\
$L(\mathrm{mH})$ & 83.2 & 2.5 \\
\hline
\end{tabular}

$f=50 \mathrm{~Hz}$, Nominal power $30 \mathrm{MVA}$

Magnetization resistance $218 \mathrm{k} \Omega$

Magnetization inductance $693 \mathrm{H}$

Table 1 and 2 show the parameters of the MV commercial electrical power lines and parameters of the threephase $66 / 20 \mathrm{kV}$ transformers respectively. The measured load parameters of each three phase series loads are in Table 3. Measurements of sending and receiving power (power loss) and voltage (voltage drop) for line 1, 2, and 3 , are shown in Table 4 .

Table 3. Measured values of the three-phase series loads

\begin{tabular}{lc}
\hline Active power & $20 \mathrm{MW}$ \\
Rective power & $6 \mathrm{MVAr}$ \\
Apparent power & $6 \mathrm{MVA}$ \\
Power factor & 0.957 \\
\hline$f=50 \mathrm{~Hz}$, Consumer voltage $18.98 \mathrm{kV}$ &
\end{tabular}

\section{Mathematical model for the simulation scheme}

We now create a simulation model and methodology for the benchmark commercial system without and with wind farm to solve the issue of voltage instability and power losses in utility networks due to the presence of electrical loads that are predominantly inductive in nature. A mathematical and simulation model of nth lines and loads is designed as our methodology. Thereafter, we apply the commercial benchmark network in our simulation model. Firstly, without wind farm (Case A) and secondly, with wind farm (Case B). This is illustrated in Fig. 2.

\subsection{Simulation scheme of the commercial system with and without wind farm (case A, case B)}

To create a simulation scheme with the proposed methodology, we carried out simulation of the commercial system without wind farm (Case A) and with wind farm (Case B) as can be seen in Fig. 3. The wind farm is made up of three wind turbines, rated at $3 \times 10=30$ MW, $3 \times 10 / 0.9=33.33$ MVA, $66 \mathrm{kV}$. In creating Case $\mathrm{A}$, the reactive loads were increased in steps of 5 , step 1 is when the commercial Case is switched off, while for steps 2 to 5 the wind farm was switched off. And Case $\mathrm{B}$ is created, with the reactive load being increased in \% values. Case B can be likened to Case A, only that the wind farm is switched $\mathrm{ON}$ from step 2 to step 5 , also the values of $Q_{\text {ref }}$ is varied from step 2 to step 5 in order to produce reactive power from the wind farm according to the usage of reactive power by the load. For Case B, the reactive power is produced according to $Q_{\text {ref }}$ and the wind speed with relation to the active power is measured and presented in Fig. 4.

For both Case A and B, we created the loads by increasing the reactive power and also $Q_{\text {ref }}$ of the wind farm. Only that for Case A, the wind farm is swithed OFF for every steps observed and measured, but for Case $\mathrm{B}$, the wind farm is only swithed OFF for step 1 and then swithed ON for step 2 to step 5. Here, the results show that in the MV transmission lines, the infused reactive power has been utilized to maintain voltage levels of the lines, so that active power can flow to do useful work on the network.

Figure 5 shows that the substation voltage decreases for Case A and there is a slight increase in the substation voltage for Case B as we move from step 1 to step 5. Also,

Table 4. Measured parameters of the benchmark commercial three-phase lines

\begin{tabular}{cccccccc}
\hline Line & $\begin{array}{c}P_{s} \\
(\mathrm{MW})\end{array}$ & $\begin{array}{c}P_{r} \\
(\mathrm{MW})\end{array}$ & $\begin{array}{c}Q_{s} \\
(\mathrm{MVAr})\end{array}$ & $\begin{array}{c}Q_{r} \\
(\mathrm{MVAr})\end{array}$ & $\begin{array}{c}U_{s} \\
(\mathrm{kV})\end{array}$ & $\begin{array}{c}U_{r} \\
(\mathrm{kV})\end{array}$ & $\begin{array}{c}P \\
(\mathrm{MW})\end{array}$ \\
1 & 16.89 & 16.45 & 7.11 & 6.41 & 64.67 & 61.38 & 0.44 \\
2 & 16.78 & 16.26 & 7.08 & 6.33 & 64.67 & 61.09 & 0.52 \\
3 & 16.66 & 16.09 & 7.02 & 6.27 & 64.67 & 60.87 & 0.57 \\
\hline
\end{tabular}




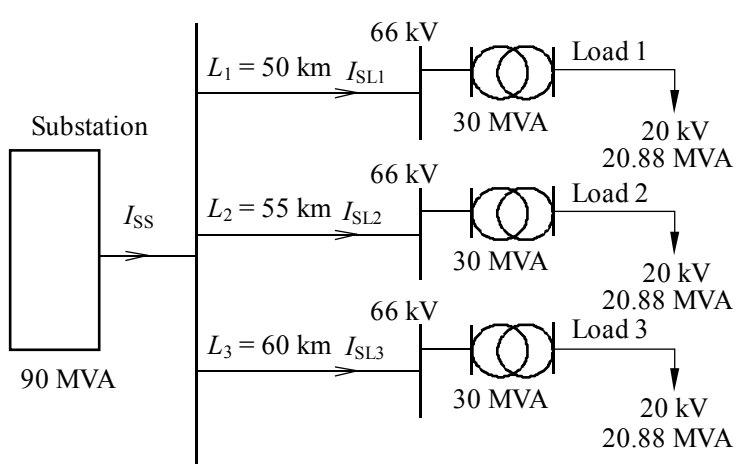

Fig. 2. Simulation model and methodology diagram without wind farm (Case A) and with wind farm (Case B)

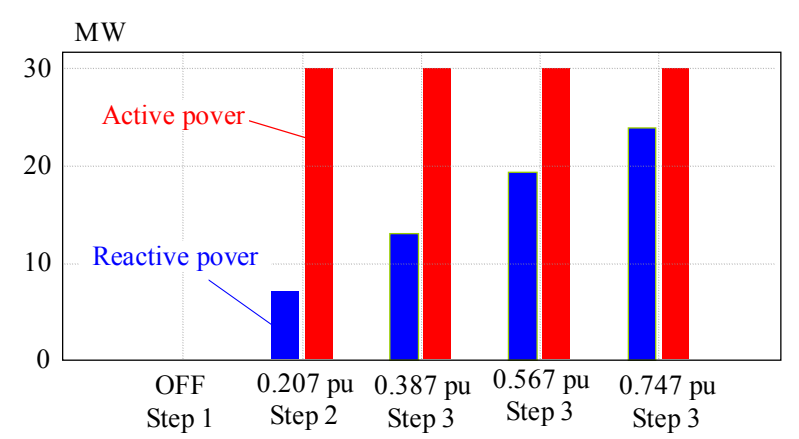

Fig. 4. Values of active and reactive power of the various steps measured from the wind farm for Case B

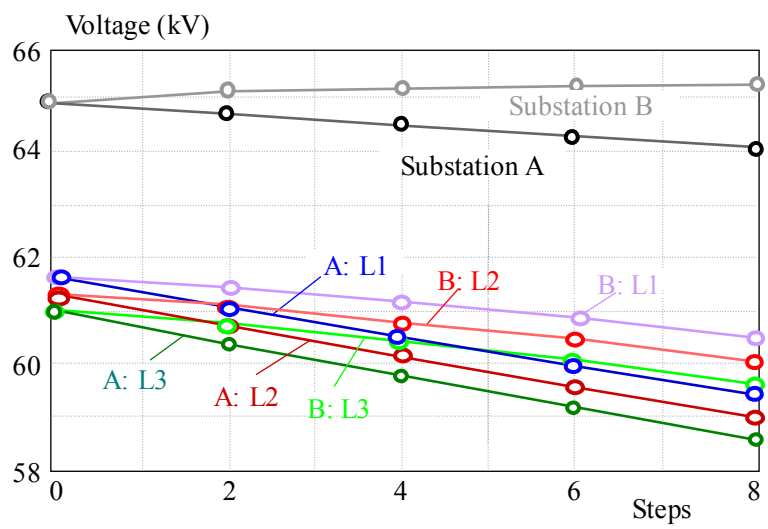

Fig. 5. The measured sending and receiving voltage levels

it is observed that there is a slight decrease in receiving voltage for lines 1, 2, and 3, for Case A and B as we move from step 1 to step 5 . This depicts that reactive power have been compensated by injecting it onto the network by virtue of the installation of the wind farm. Therefore, we can say that power losses have been reduced on the network as a result of reactive power compensation near the loads by the wind farm.

To establish the validity of the proposed scheme. The method utilized in this research work is improved upon, points 1 and 2 which are the line current of line 1 step

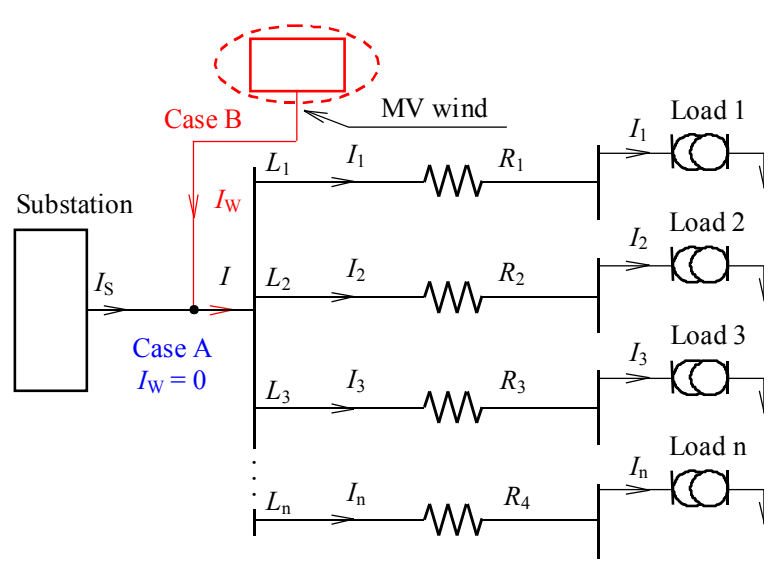

Fig. 3. Scheme of the proposed commercial (Case A) and wind farm (Case B) simulation network

5 for Case A and B respectively were chosen. The measured line currents and the real resistance value of line one $R_{1 \mathrm{~L} 1}=5.765 \Omega=5.765$ is applied to the vector equations of the proposed system to calculate power losses. The following results were gotten: Step -5, Case A , $I_{L}$ $=179.14 \mathrm{~A}$, and Step -5 , Case B, $I_{L}=182.72 \mathrm{~A}$. The results obtained in this section is the same as simulation results of the power losses of line 1 at the same points. Insinuating that the proposed scheme is valid.

The calculated values of changes in sending active power through lines 1, 2, and 3 for Case A and B is shown in Fig. 6. It can be seen that the change in sending active power for every step is higher for Case B than for Case A, and reduces as we move from step 2 to step 5. Also, for step 1 changes in sending active power for Case A and B is the same for line 1,2, and 3. Another key observation is that for Case A step 3, the change in sending active power increases as we move from line 1 to line 2, but thereafter reduces as we move from line 2 to line 3 . Overall, there is a reduction of reactive power. Also, Fig. 7 shows the values of the calculated changes in receiving active power through lines 1, 2, and 3 for Case A and B. It is observed that the change in receiving active power for every step is higher for Case B than for Case A, and reduces as we move from step 2 to step 5 . And for step 1 change in receiving active power for Case A and B is the same for line 1,2 , and 3 . The overall decrease in the sending and receiving active power in Fig. 6 and Fig. 7 depicts that the true power consumed by consumers load is very much less than the kVA capacity of the electric-power substation. Hence, even though the generators might be fully loaded, the full load of true power will not be delivered. But with reactive power infused on the network by the wind farm, the capacity of the proposed electric-power system has improved.

The values of the calculated changes in reactive power sending through lines 1,2 , and 3 for Case A and B is illustrated in Fig. 8, the change in sending reactive power rises progressively as we move from step 2 to step 5 and it is constant for line 1, 2, and 3 for every step calculated from step 2 to 5 . Furthermore, for step 1 the calculated changes in the sending reactive power for Case A and B 


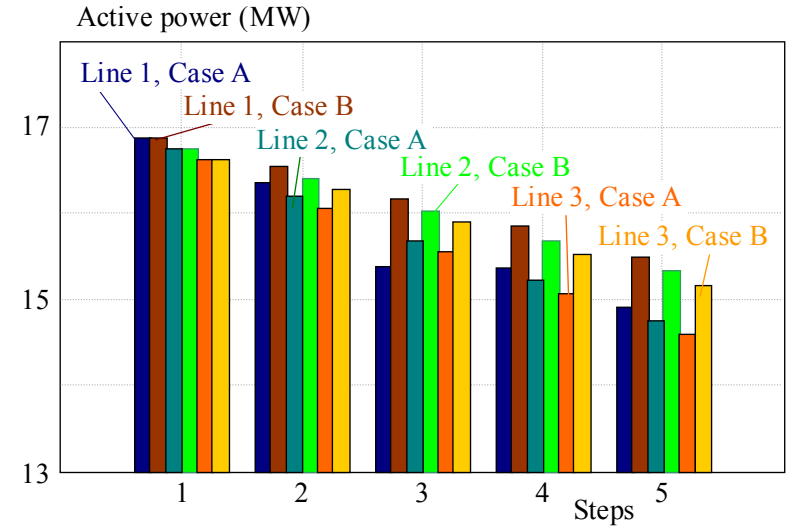

Fig. 6. Change in sending active power through Line 1, 2, and 3 for Case A, B (MW)

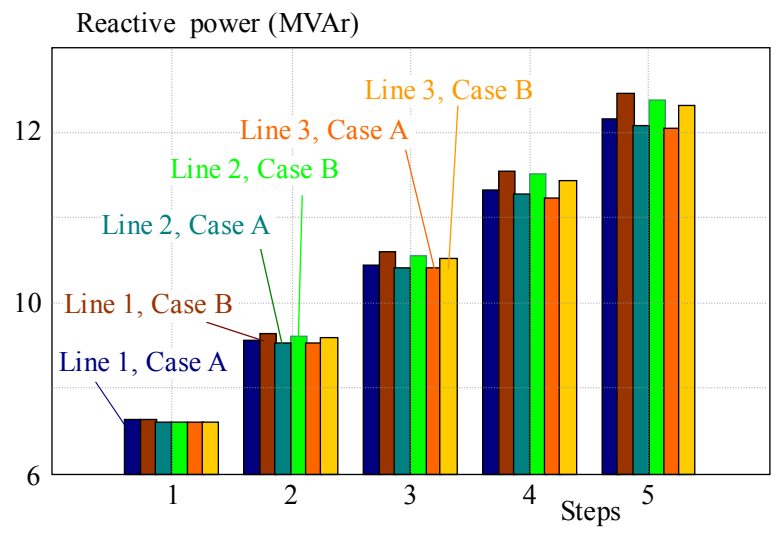

Fig. 8. Change in sending reactive power through lines for Case A and B (MVAr)

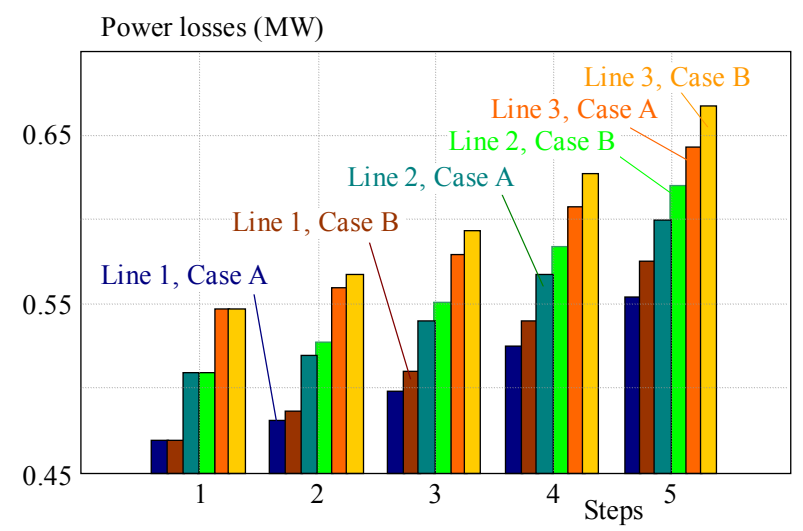

Fig. 10. Line power losses for Case A and Case B, both in MW

is the same for line 1,2 , and 3 respectively. Using the measured values of receiving reactive power for line 1,2 , and 3 for Case $\mathrm{A}$ and $\mathrm{B}$, the values of changes in receiving reactive power is calculated and tabulated in Fig. 9. The results show that for each step value calculated from step 1 to step 5, there is a slight reduction in the change of reactive power value as we move from line 1 to line 3 for Case A and B observed. Also, the line power losses for Case A and B is calculated and graphically represented in Fig. 10, the results obtained shows an increase in power losses for each step in both Cases. For step 1 the power

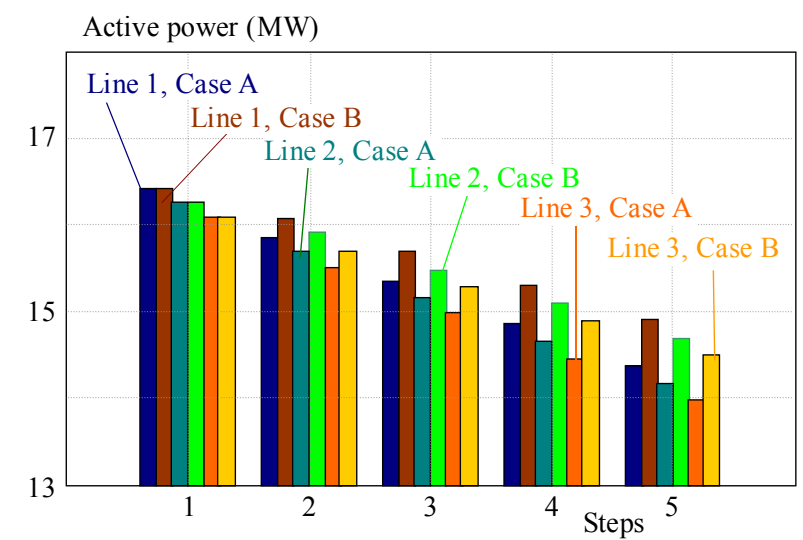

Fig. 7. Change in receiving active power through Line 1,2 , and 3 for Case A, B (MW)

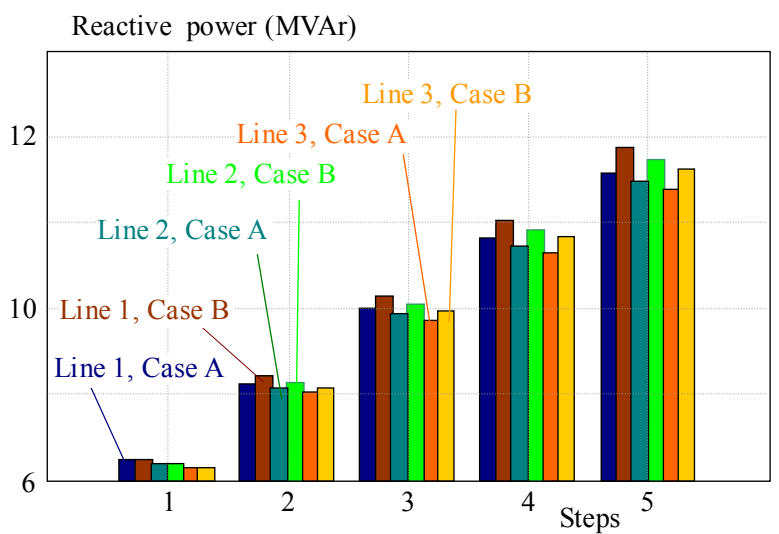

Fig. 9. Change in receiving reactive power through Lines for Case $\mathrm{A}$ and $\mathrm{B}$ (MVAr)

losses for the lines is equal for each line. While for step 2 to step 5 the power losses for line 1, 2, and 3 increases progressively as we move from line 1 to line 3 . An increase in the injected reactive power into the electrical energy supply grid results in a considerably higher absorption of current than results from simply transferring active power as illustrated in Fig. 8 and 9. This in turn results in unnecessary losses in the power transmission lines as shown in Fig. 10.

\section{Analysis of simulation results}

Analysis of power losses considering lines 1, 2, and 3 according to each step reference of step 1, for Case A and $\mathrm{B}$ is illustrated in Fig. 11, it shows that power losses increase as we move from step 2 to step 5, But for each step observed, there is a decrease in power losses as we move from step 3 to step 5 . But step 2 shows a deviation from this trend as the observed power losses decrease as we move from line 1 to line 2 and thereafter increases as we move from line 2 to line 3 . Results from step 2 is insignificant as compared to that of steps 3, 4, and 5. Overall, we can say that according to each step reference of step 1 , that there is a decrease in power losses as we move from 


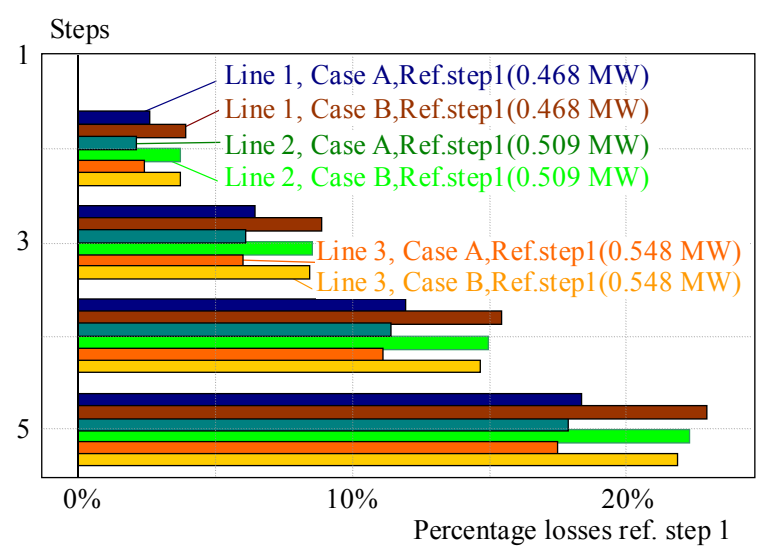

Fig. 11. Power losses of line 1, 2, 3 according to each step reference of step 1, for Case A, B (\%MW)

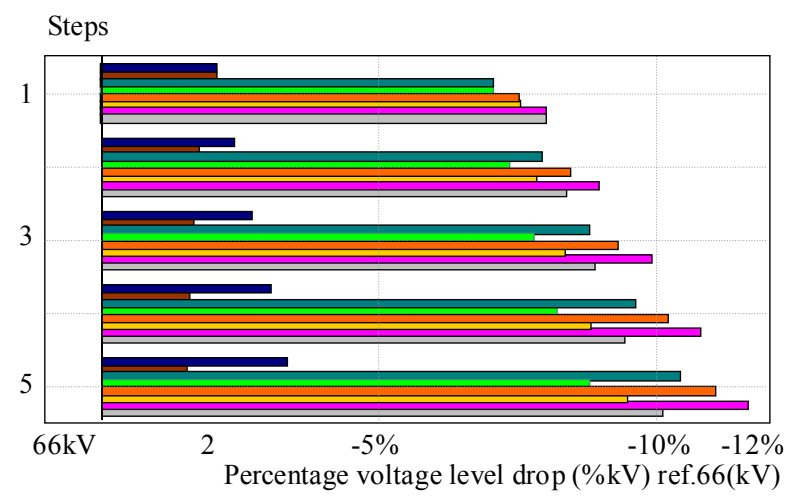

Fig. 13. The percentage voltage drop on the $66 \mathrm{kV}$ Line

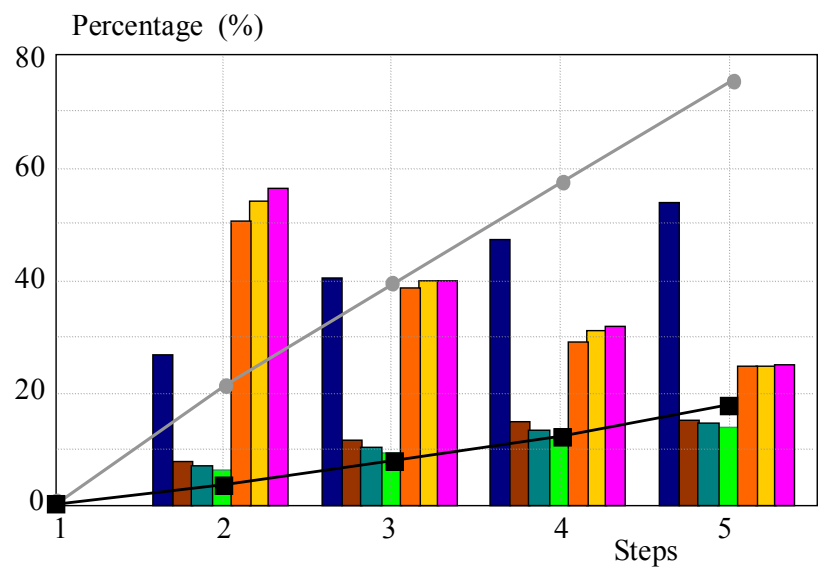

Fig. 15. Ratio of power losses and differences in voltage drop between Case A and Case B

step 2 to step 5. Although losses are inherent in distribution electricity system and cannot be eliminated. Here, increasing power system loads has brought about an increase in power losses of the proposed network. Power consumption of customer varies throughout the day and over seasons. Load is the primary driver in the MV distribution network power losses, keeping power consumption more balanced throughout the day will lower peak power loss and overall energy losses of our proposed network.

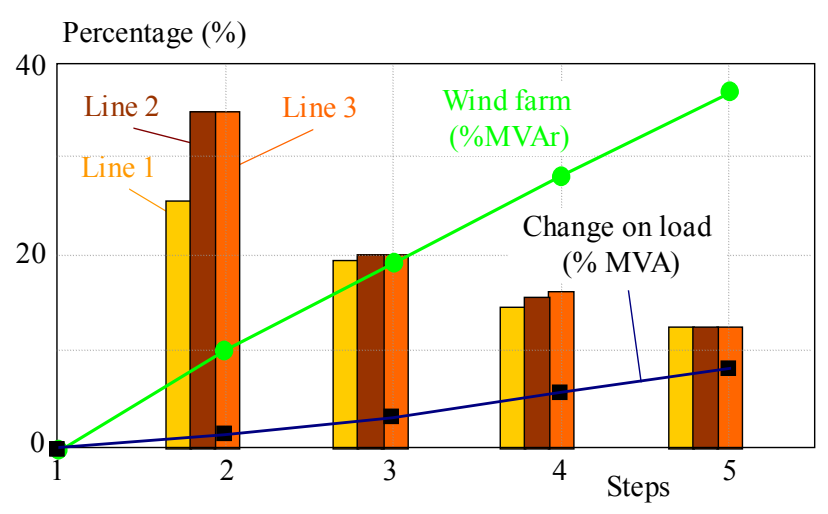

Fig. 12. Ratio of percentage power losses of each step reference of step 1 for Case A and B and the \% change in active and reactive power for the wind farm

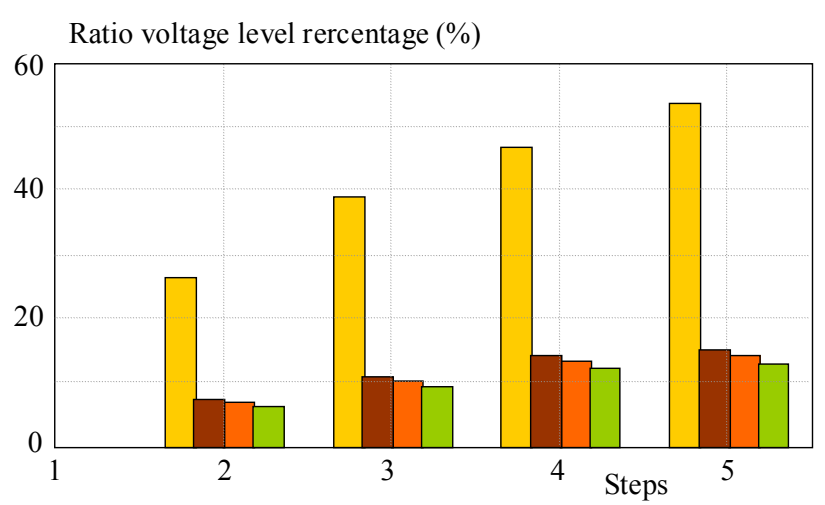

Fig. 14. Ratio of voltage level percentage on Line 1, 2, and 3 with regards to step 1 , for Case $A$ and $B$

Figure 12, reveals that the ratio of percentage losses between Case B and A of reference step 1 for line 1, 2, and 3 decreases as we move from step 2 to step 5 . But there is an increase in the percentage change of active and reactive power of the wind farm. All in all, it can be said that there is a reduction of power losses in the proposed simulation scheme. That is to say that the ratio of power losses has been minimized by installing the wind farm with IGBT power electronics converters, which was able to regulate the generated reactive power according to load usage.

Figure 13 depicts the $\%$ voltage drop within the range of + or $10 \%$ on the $66 \mathrm{kV}$ line substation and at the ends of lines 1, 2, and 3 (that is voltage receiving ends of lines 1,2, and 3). It is discovered that for Case A, sending and receiving voltage is minimized with regards to the 66 $\mathrm{kV}$ line, there was more voltage drop and power losses when considering both Cases. But comparing the values of changes in voltage looking at it the other way round with regards to Case $\mathrm{B}$ and $\mathrm{A}$, we can see that power losses have been minimized, which definitely is good, since the network is now stable, with regards to its voltage profile. It is inferred that the $\%$ voltage drop increase in Fig 13 is because of the usage of loads reactive power. In power distribution systems, a given amount of power can be transmitted with less voltage drop if a higher level 
of voltage is used. But in the proposed system infusion of reactive power has been realized using the wind farm with IGBT power electronics converters to compensate for the unwanted voltage drop.

First it is shown in Fig. 15 that the ratio of the voltage level percentage for the main substation rises as we move from step 2 to step 5 . Also, the ratio of the voltage level percentage for line 1, 2 and 3 decreases as we move from step 2 to step 5 , meaning that the voltage profile of the proposed scheme has been enhanced. Thus, when there is a decline in voltage, the current must increase to provide the same amount of power. Efficiency reduces with either a rise or fall in voltage. The existing load is a major factor in deciding how much reduction in supply voltage a reactive load can cope with. When the proposed network voltage is reduced, the current is risen to a new level, which may be more than the proposed network full-load rated ampere.

Figure 15 shows that the voltage receiving level percentage decreases on the $66 \mathrm{kV}$ line substation between Case B and A with reference to step 1 for line 1, 2, and 3 as we move from step 2 to step 5 . The percentage voltage drop on the $66 \mathrm{kV}$ Line between Case B and A with reference to step 1 increases as we move from step 2 to step 5. Also, the ratio of percentage power losses of each step reference of step 1 for Case B and A. And the \% change in active and reactive power for the wind farm increases as we move from step 1 to step 5 . Thus, it can be said that the substation encountered a fall in voltage level. The proposed wind farm distribution network undergoes some power loss and voltage drop throughout the time of transmitting power from the sending to the receiving end of our proposed network. Hence, the capability of the transmission line is decided by the wind farm systems efficiency and voltage regulation. The voltage regulation of the distribution line is a measure of changes of the receiving end voltage from no-load to full load situation from step 1 to step 5 . From the foregoing, the proposed system has relatively performed well due to the wind farms ability to efficiently regulate voltage along the distribution power lines.

\section{Conclusion}

A wind production arrangement based on gearless variable speed PMSG, MV interconnection and distribution has been proposed in this research. The new simulated system without and with a wind farm is compared, the simulated scheme without the wind farm is chosen as a representative benchmark of existing industry practice. Voltage at the receiving end of the transmission line between conditions of no load and full load were measured and expressed as a percentage of the receiving end voltage. The power obtained at the receiving end of the transmission line were generally less than the sending end power due to losses in the line resistance. Reducing voltage will not have any considerable result on the proposed system, excepting that it might assist in reducing the light load losses and ameliorate the effectiveness under this situation. It is of note to know that the efficiency of the proposed system network drops with either a high or low voltage. This study focus on the integration of a wind farm to improve the efficiency of a commercial electricity utility system over time. The efficiency of the proposed wind farm system is achieved by using higher percentage of reactive power for system generation, interconnection, transmission and distribution. The wind farm interconnection active and reactive power is permitted to be varied and regulated, in comparison with the required fixed interconnected active and reactive power for the benchmark system. Reductions in line losses have been tackled and ameliorated by installation of wind farm on the proposed distribution network. Reducing distribution line losses in the MV electric-power system is an easy obtainable alternative to enhance electric-power distribution grid efficiency. The proposed scheme results agree with other studies carried out worldwide regarding active and reactive power regulation in MV distribution network connected to a wind farm with IGBT power electronics converters.

\section{Acknowledgements}

This research has been supported by the Grant Agency of the Czech Technical University in Prague, (grant No. SGS14/188/OHK3/3T/13).

\section{REFERENCES}

[1] M. Wilch, V. S. Pappala, S. N. Singh and I. Erlich, "Reactive Power Generation by DFIG Based Wind Farms with AC Grid Connection", IEEE Power Tech, Lausanne, July 2007, 626-632.

[2] A. Ashraf, "On Reactive Power Compensation of Wind Farms Impact of Wind Farm Controller Delays", Master of Science Thesis in Electric Power Engineering, Department of Energy and Environment, Division of Electric Power Engineering, Chalmers University of Technology, Gothenburg, Sweden, June 2012.

[3] M. P. Kazmierkowski, R. Krishnan and F. Blaabjerg, "Control in Power Electronics - Selected Problems", New York, Academic, 2002.

[4] G. Fandi, J. Svec and Z. Muller, "The Converter Choice and its Control Circuit Design for Synchronous Generators", 14th International Scientific Conference on Electric Power Engineering (EPE), Kouty nad Desnou, May 2013, 697-701.

[5] F. D. Gonzialez, M. Martinez-Rojas, A. Sumper, O. Gomis-Bellmunt and L. Trilla, "Strategies for Reactive Power Control in Wind Farms with STATCOM", EPE Wind Energy Chapter Symposium, 2010, 1-10,

[6] S. M. Yousuf and S. Arthi, "Reactive Power Improvement in Wind Farm by Using UPQC", International Journal of Science and Research (IJSR) 3 no, 4 (April 2014), 403-408.

[7] M. Milligan, K. Porter, E. Demeo, P. Denholm, H. Holttinen, B. Kirby, N. Miller, A. Mills, M. O'malley, M. Schuerger and L. Soder, "Wind Power Myths Debunked", IEEE Power and Energy Magazine, 7 no. 6 (November/December 2009), 89-99.

[8] F. Blaabjerg, M. Liserre and K. Ma, "Power Electronics Converters for Wind Turbine Systems", IEEE Transactions on Industry Applications, 48 no. 2 (March/April 2012), 708-719. 
[9] G. Fandi, F. O. Igbinovia, Z. Muller, J. Svec and J. Tlusty, "Using Renewable MV Wind Energy Resource to Supply Reactive Power in MV Distribution Network", IEEE16th International Scientific Conference on Electric Power Engineering (EPE), Kouty nad Desnou, May 2015, 169-173,.

[10] G. Fandi, F. O. Igbinovia, J. Svec, Z. Muller and J. Tlusty, "Advantageous Positioning of Wind Turbine Generating System in MV Distribution Network", IEEE17th International Scientific Conference on Electric Power Engineering (EPE), Prague, May 2016.

[11] M. Liserre, R. Cardenas, M. Molinas and J. Rodriguez, "Overview of multi-MW wind turbines and wind parks", IEEE Transactions on Industrial Electronics, 58 no. 4 (April 2011), 1081-1095.

[12] F. Janicek, M. Mucha, K. Cesnek and J. Kovacik, "Controlled Switching of the T402 Transformer in the Krizovany 400kV Substation", Journal of Electrical Engineering, 61 no. 1 (January 2010), 11-19.

[13] J. Snajdr, J. Sedlacek and Z. Vostracky, "Application of A Line Ampacity Model and Its Use in Transmission Lines Operations", Journal of Electrical Engineering, 65 no. 4 (2014), 221-227.

[14] P. T. Orsgov, "Ripple Control Signal Using for Earth Fault Location in MV Networks", Journal of Electrical Engineering, 56 no. 11-12 (2005), 313-321.

[15] M. Kolcun and J. Szkutnik, "The Methodology for Optimization of Energy Distribution Network", Journal of Electrical Engineering, 60 no. 4 (July 2009) 219-222.

[16] Z. Chen, J. M. Guerrero and F. Blaabjerg, "A Review of the State of the Art of Power Electronics for Wind Turbines", IEEE Transactions on Power Electronics, 24 no. 8 (August 2009), 1859-1875.

[17] A. D. Hansen, F. Iov, F. Blaabjerg and L. H. Hansen, "Review of Contemporary Wind Turbine Concepts and Their Market Penetration", Wind Engineering, 28 no. 3 (May 2004), 247-263.

[18] F. Blaabjerg and K. Ma, "Future on Power Electronics for Wind Turbine Systems", IEEE Journal of Emerging and Selected Topics in Power Electronics, 1 no. 3 (September 2013), 139-152.

Received 30 June 2017

Ghaeth Fandi was born in Latakia, Syria. He holds a degree in Electrical Engineering from the University of Tishreen, Latakia, Syria. He also holds Master degree in Renewable Energy from the same University. And he is currently a Doctorate $(\mathrm{PhD})$ Candidate in the Department of Electrical Power Engineering at Czech Technical University in Prague, Czech
Republic. His dissertation research is focused on renewable energy dispersed generation sources in distribution system. Other activities include hybrid simulation of power systems, power quality and research of control of electrical power systems. He is a member of the Institute of Electrical and Electronics Engineers (IEEE).

Famous O. Igbinovia received his Bachelor of Engineering (BEng) degree in Electrical/Electronic Engineering from Ambrose Alli University, Ekpoma, Nigeria, the Master of Engineering (MEng) degree in Power and Machines from the University of Benin, Benin City, Nigeria, and the Master of Science (MSc) degree in Business Administration from Czech University of Life Sciences in Prague, Czech Republic. He is currently a Doctorate (PhD) Candidate in the Department of Electrical Power Engineering at Czech Technical University in Prague, Czech Republic. His research interests are, but not limited to, renewable energy systems; control of electrical power systems; power quality; distributed and dispersed generation sources in distribution systems; electricity market; and hybrid simulation of power systems. He is a member of the Institute of Electrical and Electronics Engineers (IEEE).

Josef Tlustý, is currently a Professor of Electrical Power Engineering at Czech Technical University in Prague, Czech Republic. In 1971 he obtained a Master degree in Power Engineering (Energy utilisation) from Czech Technical University in Prague, Czech Republic and in 1978 the $\mathrm{PhD}$ degree in Power Engineering (Transmission of Electrical Energy) from the same university. His research interests include; renewable energy systems, control and protection of electrical power systems, distributed generation sources in distribution systems, power voltage quality in electrical power systems and the use of Flexible Alternating Current Transmission Systems (FACTS) in electrical networks. He is a senior member of the Institute of Electrical and Electronics Engineers (IEEE).

Rateb Muhammad born in Tartus, Syria, graduated with a degree in Electrical Engineering from the University of Tishreen, Latakia, Syria in 1989. Currently, he is the Tartous Branch Chairman of Syrian Engineering Society, also he is the regional manager of generation, transmission and distribution of the Syrian ministry of electricity, his research interest includes MV electrical system, minimizing power losses and renewable energy systems. 